\title{
Erratum to: Somatostatin-Producing Atypical Null Cell Adenoma Manifesting as Severe Hypopituitarism and Rapid Deterioration-Case Report
}

\author{
Yoshikazu Ogawa • Mika Watanabe - Teiji Tominaga
}

Published online: 30 October 2010

(C) Springer Science+Business Media, LLC 2010

Erratum to: Endocr Pathol (2010) 21:130-134

DOI 10.1007/s12022-010-9110-2

To the readership: Figures were incorrectly published in the above-mentioned article (p132), although figure legends were correct.

Figure 2 must be changed as Figure 3. The legend is below. Photomicrograph of the surgical specimen showing weak but distinctive expression of somatostatin in the cytoplasm. Original magnification x 200 .

Figure 3 must be changed as Figure 2. The legend is below. Photomicrograph of the surgical specimen showing significant positive reaction to Ki-67. Staining was also seen in cytoplasm and cell membrane. Labeling index is more than $18 \%$. Original magnification x 80 .

The online version of the original article can be found at http://dx.doi. org/10.1007/s12022-010-9110-2.

Y. Ogawa $(\bowtie)$

Department of Neurosurgery, Kohnan Hospital,

4-20-1 Nagamachiminami, Taihaku-ku,

Sendai, Miyagi 982-8523, Japan

e-mail: yogawa@kohnan-sendai.or.jp

M. Watanabe

Department of Pathology, Tohoku University

Graduate School of Medicine,

Sendai, Japan

T. Tominaga

Department of Neurosurgery, Tohoku University

Graduate School of Medicine,

Sendai, Japan 